\title{
Preferential Termination of Corticorubral Axons on Spine-Like Dendritic Protrusions in Developing Cat
}

\author{
Yasuhiko Saito, Wen-Jie Song, and Fujio Murakami \\ Department of Biophysical Engineering, Faculty of Engineering Science, Osaka University, Toyonaka, Osaka 560, Japan
}

The formation of synaptic contacts is a crucial event during neural development and is thought to be achieved by complex interactions between incoming axons and the neurons in the target. We have focused on spine-like dendritic protrusions (SLDPs), which are transient pleomorphic protrusive structures seen in developing brains. Although the functional significance of SLDPs remains unknown, accumulating in vitro evidence suggests that the SLDP plays an important role in synaptogenetic interactions with axons. As a test of this idea, the present study was performed to examine whether the SLDPs are the preferential sites of synapse formation in vivo.

The ultrastructure of biocytin-labeled corticorubral (CR) terminals was examined in serial thin sections during the period of synaptogenesis in newborn cats. We found that a major pro- portion (86\%) of the CR synapses was formed on SLDPs. The presynaptic terminals were often invaginated by fine processes extending from the tips of SLDPs. Synaptic structures presumably of cortical origin were also found on SLDPs of HRP-labeled rubrospinal cells, suggesting that SLDPs postsynaptic to labeled CR terminals originate at least in part from rubrospinal cells. Taken together, these results indicate that SLDPs may represent preferred sites of synapse formation and support the notion that SLDPs play a role in synaptogenic interactions during brain development.

Key words: cat; dendritic spine; dendritic filopodia; synaptogenesis; corticorubral; rubrospinal; electron microscopy; biocytin
During brain development, growth cones navigate through a complex environment to reach their target. Recent studies have revealed various kinds of interactions during growth cone navigation (for review, see Dodd and Jessel, 1988; Goodman and Shatz, 1993; Goodman, 1996), but relatively little is known about what interactions occur within the final target. It is presumed that a cascade of complex events must take place at the target, because not only the presynaptic axons but also the postsynaptic cells must be continuously growing and remodeling (for review, see Jacobson, 1991). Among these events, one of the most important is the interaction associated with synaptogenesis. In this context, dendritic filopodium- or spine-like dendritic protrusions (SLDPs) as well as dendritic growth cones have attracted considerable attention. SLDPs emanate from the dendritic shaft and show pleomorphic morphological features, including filiform structures distinct from the dendritic spines in adults (e.g., Morest, 1969; Scheibel et al., 1973). They also transiently increase in number at an early stage of development (e.g., Morest, 1969; Scheibel et al., 1973; Lund et al., 1977; Boothe et al., 1979; Garey and Saini, 1981; Hammer et al., 1981; Phelps et al., 1983; Dvergsten et al., 1986; Ramoa et al., 1988). These in vivo findings suggest that SLDPs are continuously remodeled in development.

\footnotetext{
Received June 16, 1997; revised Aug. 25, 1997; accepted Aug. 28, 1997.

This work was supported by Ministry of Education, Science and Culture of Japan Grant-in-Aid 07279101. We thank Hironobu Katsumaru, Yoichi Oda, Edward Ruthazar, Ryuichi Shirasaki, and Michael Spooner for critically reading this manuscript, Hironobu Katsumaru, Tong-Hang Go, and Kouichi Hashimoto for partial participation in the experiment, Tadashi Isa for continuous encouragement, Masanori Kanda for supplying the experimental animals, Hiroshi Maebashi for assistance in image analysis, and Kyoko Katayama for secretarial assistance.

Correspondence should be addressed to Fujio Murakami, Department of Biophysical Engineering, Faculty of Engineering Science, Osaka University, Toyonaka, Machikaneyama 1-3, Osaka 560, Japan.

Dr. Saito's present address: National Institute for Physiological Sciences, Myodaiji-chou, Nishigou-naka, Okazaki 444, Japan.

Copyright (C) 1997 Society for Neuroscience $0270-6474 / 97 / 178792-12 \$ 05.00 / 0$
}

Time-lapse studies of developing hippocampal neurons in vitro have demonstrated that SLDPs are rapidly remodeled by protrusive activity (Dailey and Smith, 1996; Ziv and Smith, 1996). The in vitro observation that SLDPs initiated contacts with axons, leading to the formation of presynaptic bouton-like structures, raised the possibility that SLDPs actively initiate synaptogenetic contacts with axons (Dailey and Smith, 1996; Ziv and Smith, 1996). Consistent with this idea is the finding that synapses occur on filopodial dendritic protrusions in developing brains (Saito et al., 1992).

If the notion that SLDPs play a crucial role in the formation of synapses on the dendrite is correct, SLDPs should be sites of termination for incoming axons. In addition, a specialized synaptic morphology might occur between incoming axons and SLDPs. These notions can be tested in the most straightforward manner by quantitative electron microscopy of identified synaptic profiles.

The present study was performed to analyze quantitatively whether incoming axons form synaptic contacts on SLDPs in a specific manner. To achieve this aim, we examined biocytinlabeled corticorubral (CR) synapses of newborn cats in threedimensional reconstructions of serial thin sections. We have chosen the CR system of the cat, because there are abundant data about the synaptic organization of the feline red nucleus (RN) (Tsukahara and Kosaka, 1968; Nakamura and Mizuno, 1971; Pizzini et al., 1975; Tsukahara et al., 1975; Nakamura et al., 1978; Murakami et al., 1982, 1983, 1986; Katsumaru et al., 1984) and the development of its afferents (Villablanca et al., 1982; Tsukahara et al., 1983; Kosar et al., 1985; Murakami and Higashi, 1988; Higashi et al., 1990; Murakami et al., 1991a,b, 1993; Song et al., 1995a); particularly, it is well established that CR fibers in the adult cat rubrospinal cells terminate on the dendritic membrane remote from the soma (Tsukahara and Kosaka, 1968; Tsukahara et al., 1975; Murakami et al., 1982). 
In the present study, we show that a major proportion of the CR synapses in newborn cats is formed on SLDPs, presumably originating from rubrospinal cells. These results suggest that SLDPs of rubrospinal cells represent preferred sites of synapse formation for cortical inputs.

\section{MATERIALS AND METHODS}

Labeling of corticorubral fibers and rubrospinal cells. Seven kittens at 2-5 postnatal days were obtained from a breeding colony of Aburahi Labs (Shionogi and Co., Ltd., Shiga, Japan). Newborn kittens were used, because extensive synaptogenesis of the CR inputs appears to occur during the first postnatal month (Higashi et al., 1990; Song et al., 1995a). For injection of biocytin, a glass micropipette with a tip diameter of $40-50 \mu \mathrm{m}$ was connected to a Hamilton syringe with a polyethylene tube. Biocytin (Sigma, St. Louis, MO; 5\% in Tris buffer) was pressure-injected into the sensorimotor cortex under anesthesia with sodium pentobarbital (Nembutal; $20 \mathrm{mg} / \mathrm{kg}$, i.p.). The unilateral injection totaling $0.5-1.5 \mu \mathrm{l}$ in each animal was made at one to three loci of the pericruciate cortex; biocytin was injected at two depths $(1.5$ and $1.0 \mathrm{~mm}$ from the pial surface) per locus, $0.25 \mu$ l per depth.

The procedure for intracellular staining in newborn cats was detailed previously (Song et al., 1995b). HRP was intracellularly injected into physiologically identified rubrospinal cells by iontophoresis with a glass microelectrode filled with 5-8\% HRP (grade II; Toyobo, Osaka, Japan). The injection was made by passing $550-800 \mathrm{msec}$ positive current pulses of 2.0-5.0 nA followed by $40-60 \mathrm{msec}$ negative pulses of $0.5-1.0 \mathrm{nA}$ at $0.8-1.5 \mathrm{sec}$ intervals for $10-45 \mathrm{~min}$. One to three rubrospinal (RS) cells were injected in each of four animals that had also been injected with biocytin.

Histological procedures. Immediately after the HRP injection (or $3 \mathrm{~d}$ after the biocytin injection in animals that had not received an HRP injection), the kittens were transcardially perfused with a mixture of $1 \%$ paraformaldehyde and $1 \%$ glutaraldehyde followed by $6 \%$ glutaraldehyde under deep anesthesia with Nembutal $(>35 \mathrm{mg} / \mathrm{kg}$, i.p.). This was followed by a postwash with $50 \mathrm{~mm}$ PBS (pH 7.4) in the HRP-injected animals. The brain was then dissected and kept in $6 \%$ glutaraldehyde or PBS overnight. A brain block including the RN was trimmed and cut horizontally into sections with a Microslicer (Dosaka EM, Kyoto, Japan) at $50-80 \mu \mathrm{m}$.

Sections containing the $\mathrm{RN}$ were first incubated with $0.05 \% 3,3^{\prime}$ diaminobenzidine tetrahydrochloride (DAB) for $30 \mathrm{~min}$ followed by reaction with a mixture of DAB and $0.015 \% \mathrm{H}_{2} \mathrm{O}_{2}$ for $30 \mathrm{~min}$ at room temperature. Then the sections were processed according to the goldsubstituted silver peroxidase intensification method (van den Pol and Gorse, 1986) with a slight modification. In brief, the sections were incubated in thioglycolic acid for $4 \mathrm{hr}$ and then reacted for silver intensification for $8 \mathrm{~min}$. This procedure produced fine granular reaction products, which permitted the discrimination of the HRP-injected cells from the biocytin-incorporated CR fibers, which had not undergone the intensification procedure.

To visualize the CR fibers, the sections were permeabilized with $0.05 \%$ Triton $\mathrm{X}-100$ in $50 \mathrm{~mm}$ Tris-buffered saline ( $\mathrm{pH} 7.6)$ and processed using the avidin-biotin-peroxidase complex method (Vector Laboratories, Burlingame, CA). The sections were post-fixed in $2 \%$ $\mathrm{OsO}_{4}$, stained en bloc with $1.5 \%$ uranyl acetate, dehydrated, infiltrated in propylene oxide, and then flat-embedded in Epon (TAAB, Berkshire, England).

Electron microscopy. Fibers were sampled from the magnocellular region of the RN (RMG), which was easily discerned by its darker appearance under the light microscope (Fig. 1B). Epon blocks $(\sim 500 \times$ $500 \mu \mathrm{m}$ ) including biocytin-labeled fibers were trimmed out from the RMG ipsilateral to the injection site. One block near the center of the RMG or two blocks each from the medial and the lateral parts of the RMG were picked out from three to six sections. In total, four to six blocks were thus obtained from each animal. The block was attached to the top of a cylindrical Epon block and further cut into $5 \mu \mathrm{m}$ sections. The $5 \mu \mathrm{m}$ sections were coverslipped with Epon. After detailed light microscopic observations, sections containing labeled fibers with several axonal swellings were arbitrarily chosen and photographed. The 5- $\mu \mathrm{m}-$ thick sections were reattached to other cylindrical Epon blocks. Blocks were trimmed to center the CR fibers that had been chosen in the preceding light microscopic observation and then rephotographed for electron microscopic analysis. They were then cut into ultrathin sections with an ultramicrotome (Reichert-Jung Ultracut E), collected on
Formvar-coated single-slot grids, and observed with an electron microscope (1200EX; Jeol, Tokyo, Japan). The light micrographs were referred to to find the corresponding profiles under the electron microscope. To examine whether the selection of axonal swellings described above caused a sampling bias, one $50-\mu \mathrm{m}$-thick Epon block containing biocytin-labeled fibers was directly cut into ultrathin sections and collected on single-slot grids for electron microscopy.

HRP-labeled RS cells were drawn using a drawing tube attached to a light microscope (BH2; Olympus, Tokyo, Japan) and reconstructed from 50- to $80-\mu \mathrm{m}$-thick serial sections. The sections were then cut into 5-8 $\mu \mathrm{m}$ sections and processed as described above.

Identification of synapses. Profiles with parallel membranes at putative presynaptic and postsynaptic terminals and dense material in the synaptic cleft were regarded as synapses if they further satisfied one or both of the following criteria (see Vaughn, 1989): (1) synaptic vesicles accumulating at the presynaptic membrane specialization, and (2) postsynaptic membrane specialization and thickening. The presence of synapses was confirmed by observing at least three consecutive sections.

Synapses on HRP-labeled dendrites were often obscured by dense DAB reaction product and, therefore, did not satisfy the latter criterion. However, parallel membranes between the presynaptic and postsynaptic plasma membranes with dense material in the cleft and an accumulation of synaptic vesicles toward the presynaptic membrane could be recognized. Approximately $10 \%$ of the CR synapses did not exhibit an obvious postsynaptic membrane specialization. It is likely that these represent primordial synapses (Hayes and Roberts, 1973; Hinds and Hinds, 1976; Juraska and Fifkova, 1979; Blue and Parnavelas, 1983; Kunkel et al., 1987; Vaughn, 1989). These synapses were, therefore, included in the analysis, although they did not satisfy the latter criterion.

Any postsynaptic profiles containing microtubules were regarded as dendritic shafts, and dendritic protrusions lacking microtubules and with cytoplasmic features described below (see Results) were regarded as SLDPs.

Three-dimensional reconstruction from serial sections. The outlines of the presynaptic and the postsynaptic profiles were traced on sheets of semitransparent paper overlaid on electron micrographs. The tracings were then captured through a video camera onto a hard disk using an image-processing device (Olympus TVIP-5100) and reconstructed and edited using a three-dimensional reconstruction program (TRI programs; Ratoc System, Tokyo, Japan).

Quantitative analysis. Three kittens injected with biocytin but not HRP were used for quantitative analysis of synaptic loci on the soma-dendritic membrane of RN cells. Biocytin-labeled fibers in 5- $\mu \mathrm{m}$-thick Epon blocks were cut into serial thin sections. In total, 41 blocks were analyzed, and the total length of the axons analyzed was $4.8 \mathrm{~mm}$. A single presynaptic bouton sometimes formed synapses with multiple postsynaptic profiles; in such cases, the number of synapses was counted as 1 irrespective of the number of active zones formed by the presynaptic bouton.

To estimate the length of SLDPs, their outlines were traced from electron micrographs of serial sections onto sheets of semitransparent paper, which were then superimposed to obtain a two-dimensional projection of the dendritic protrusions. The distance from the dendritic shaft to the ending of the protrusion was then measured to give its length. SLDPs on the HRP-labeled RS cells extending from dendrites $<100 \mu \mathrm{m}$ from the soma were chosen for analysis, because most CR synapses terminated on SLDPs in this region (see Fig. 8). The diameter of the parent dendritic shaft, $D$, was estimated from the equation, $D=2$ $\sqrt{ }(A / \pi)$, where $A$ is the cross-sectional area of the dendritic shaft. When the dendrites were cut parallel to their longitudinal axis, the diameter was defined as the maximum length perpendicular to the axis.

Three HRP-labeled RS cells from three kittens were used to estimate the relation between the diameter of the dendritic shaft and the distance from the soma of HRP-labeled RS cells. Five to seven dendrites were selected arbitrarily from each cell and reconstructed with a $60 \times$ objective with the aid of a Neuron Tracing System (Eutectic Electronics, Inc., Raleigh, NC). The ratio of the dendritic surface area occupied by the SLDPs and the shafts was estimated from two dendritic fragments of HRP-labeled RS cell dendrites $\sim 2 \mu \mathrm{m}$ in diameter (see Fig. 8). From a series of electron micrographs taken from serial sections of the dendritic fragments, the circumferences of the SLDP cross-sections and of the dendritic shafts were measured. Then the ratios between the total lengths of the circumferences of the SLDP cross-sections and those of the dendritic shafts were calculated for each dendritic segment. These ratios should approximate the ratios of the surface area of the SLDP to that of dendritic shafts. The total length of the dendrites analyzed was $33 \mu \mathrm{m}$. 

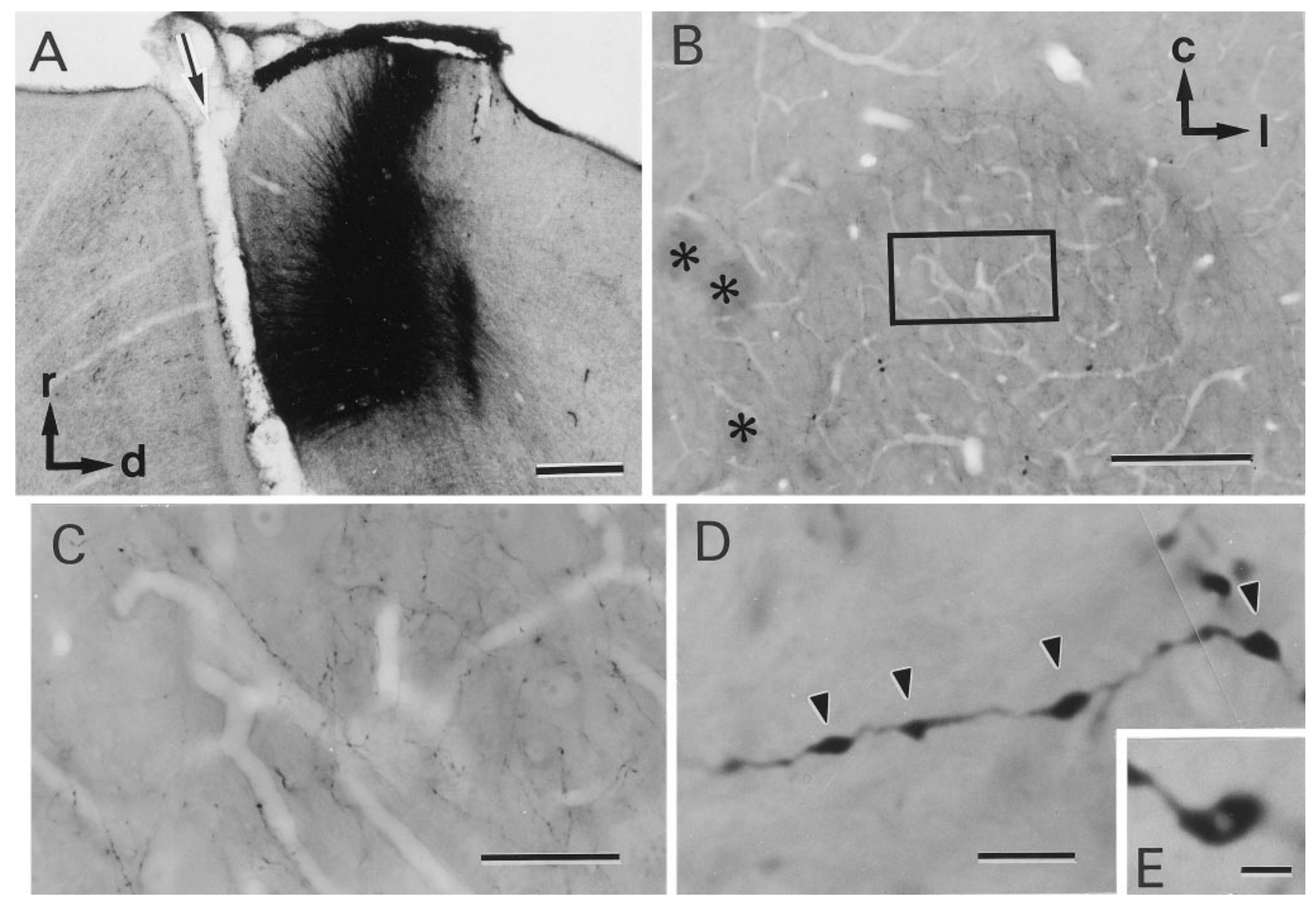

Figure 1. Identification of corticorubral fibers. $A$, Injection site of biocytin. A sagittal section of the sensorimotor cortex is shown. The arrow indicates the cruciate sulcus. $r$, Rostral; $d$, dorsal. $B$, Low-magnification photomicrograph of a horizontal section of the RN. Asterisks indicate the oculomotor nerve. $c$, Caudal; $l$, lateral. $C$, High-magnification photomicrograph of the area of the RN outlined by the rectangle in $B$. Many biocytin-labeled fibers can be seen. $D, E$, Higher-magnification photomicrographs of biocytin-labeled fibers. Axonal swellings are seen along the fibers (arrowheads). Some of the axonal swellings were fenestrated, as shown in $E$. Scale bars: $A, 500 \mu \mathrm{m} ; B, 200 \mu \mathrm{m} ; C, 50 \mu \mathrm{m} ; D, 4 \mu \mathrm{m} ; E, 2 \mu \mathrm{m}$.

All surgery and procedures on animals followed the guidelines of animal experiments approved by the Committee of Osaka University on Animal Research.

\section{RESULTS}

\section{Light microscopic appearance of CR fibers}

Injection of biocytin was restricted to the sensorimotor cortex, as shown in Figure $1 A$. Labeled fibers coursed through the internal capsule and extended through the cerebral peduncle. In the RMG region, numerous labeled fibers were observed in contrast to surrounding regions (Fig. $1 B, C$ ). These fibers were generally thin $(<0.1 \mu \mathrm{m})$ and infrequently bifurcated (Fig. $1 C)$ and often ended in growth cones. Short side branches were occasionally seen (data not shown). Axonal swellings, 0.1-1 $\mu \mathrm{m}$ in diameter, were observed along the course of the fibers (Fig. 1D, arrowheads), occasionally exhibiting a lighter region in their centers (Fig. 1E).

\section{Synaptic structure of CR axons}

Electron microscopic observations of labeled fibers with various light microscopic morphological features revealed that synapses were mostly associated with axonal swellings. As shown in the electron micrographs of Figures 2 and 3, CR fibers often formed synapses on SLDPs extending from a dendritic shaft (Fig. 2). These SLDPs often protruded into the labeled axonal swellings and were sometimes encapsulated by the swellings (Fig. 3A-D); such invaginated structures were found to correspond to axonal swellings with a lighter central region under the light microscope (Fig. 1E). Three-dimensional reconstruction of CR synapses revealed that dendritic protrusions sometimes branched in a complicated manner (Fig. 3E). Unlabeled axon terminals were also found to be invaginated by dendritic protrusions (data not shown).

The cytoplasm of SLDPs usually contained vesicular structures (Figs. 2, 3, arrowheads). The SLDPs were frequently associated with smooth endoplasmic reticulum (Fig. 2A,B, small arrows), occasionally with multivesicular bodies, but the spine apparatus (Peters et al., 1991) was rarely observed. Mitochondria were occasionally found, most of them being restricted to the necks or proximal parts of the protrusions (Fig. 3). Polyribosome-like granules were also observed in the heads or tips of the protrusions (data not shown).

\section{Localization of CR synapses}

To examine the localization of CR synapses on the soma-dendritic membrane of RN cells, a quantitative analysis was performed. In total, 76 synapses were analyzed from sets of serial sections cut from 5- $\mu$ m-thick sections in three kittens injected with biocytin but not HRP. In all of the three kittens, most of the synapses were found on SLDPs (Fig. $4 A-C$ ). Moreover, a similar result was obtained from a block that was directly cut into thin sections (Fig. 4D), suggesting that selection of axonal swelling- 

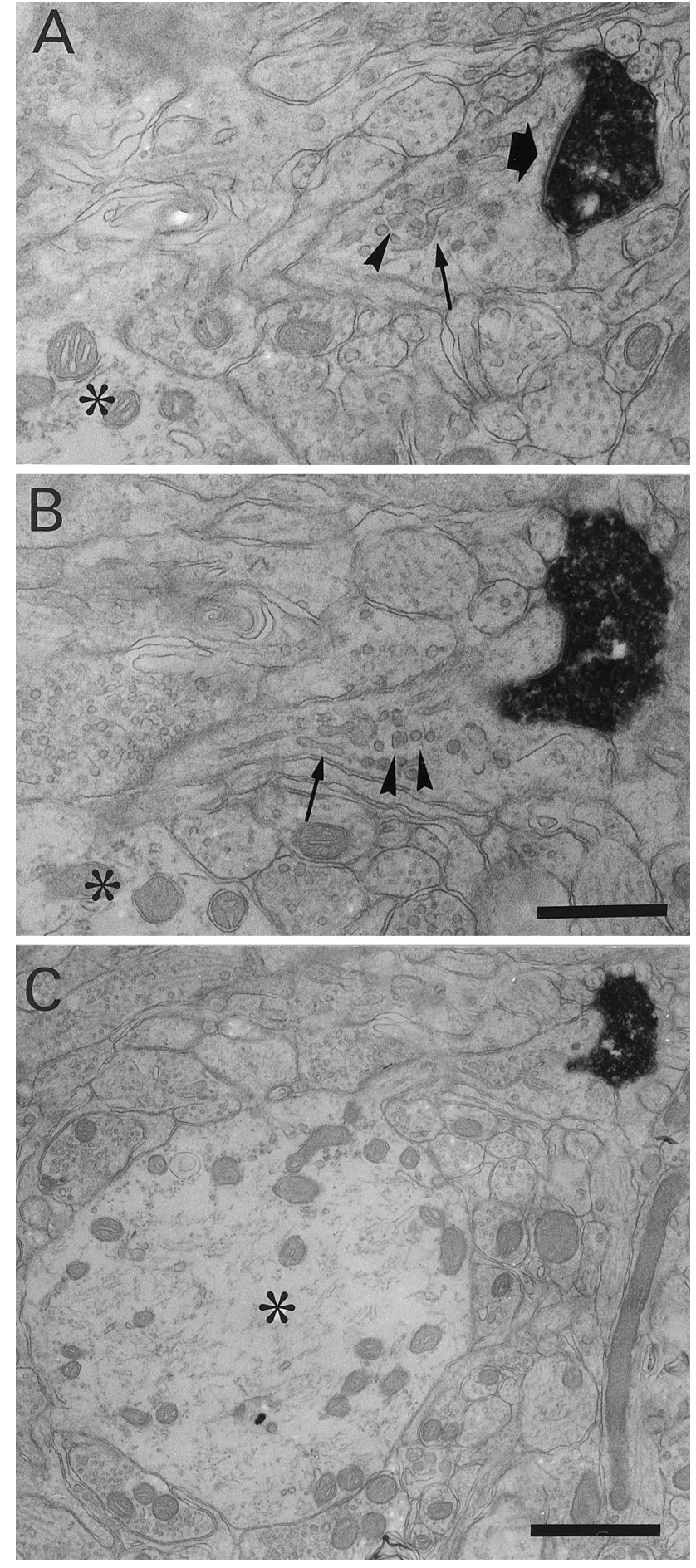

Figure 2. Biocytin-labeled CR synapse on an SLDP. $A, B$, Electron micrographs from semiserial sections of a CR fiber. The CR fiber formed synapses (black arrow) on an SLDP emanating from a dendritic shaft (asterisk). SLDPs often contained vesicular structures (arrowheads) and smooth endoplasmic reticulum (small arrows). C, Low-magnification photomicrograph showing a filiform process emerging from a dendritic shaft (asterisk). Scale bars: $A, B, 0.5 \mu \mathrm{m} ; C, 1 \mu \mathrm{m}$.

rich $5 \mu \mathrm{m}$ sections (see Materials and Methods) did not affect the result. Eighty-six percent of the CR synapses analyzed in newborn kittens occurred on SLDPs. Of these CR synaptic endings on SLDPs, $\sim 35 \%$ (36 of 103 synapses) were invaginated by
SLDPs, forming complex synaptic structures. The length of SLDPs on which CR synapses were formed ranged from 0.28 to $3.87 \mu \mathrm{m}$, with a mean of $1.10 \mu \mathrm{m}(n=31)$. Together, these findings indicate that most CR fibers in newborn cats form synapses on SLDPs.

\section{Dendritic protrusions on RS cells}

Next we analyzed the morphology of RS cell dendrites and the synapses on these dendrites to determine whether SLDPs that bear CR synapses originate from RS cells. HRP-filled RS cells in newborn cats elongated dendrites, up to $500 \mu \mathrm{m}$ in length, infrequently ending in growth cone-like bulbous endings (data not shown). As shown in Figure 5, numerous SLDPs, up to $2 \mu \mathrm{m}$ in length, emanated from these dendritic shafts extending from RS cells. The protrusions were notable on proximal dendrites and those somewhat remote from the soma; they varied in both length and shape; some exhibited filiform shapes, whereas others had bulbous heads. Electron microscopic observation confirmed that synapses were formed on RS cell SLDPs (Fig. 6). As shown in the high-power electron micrographs of Figure $6, C$ and $D$, synapses often occurred on HRP-labeled SLDPs (Fig. 6C,D, arrows), which in some cases branched in a complicated manner. Synapses were also found on SLDPs extending into presynaptic axonal terminals (Fig. 7). The length of the SLDPs that received synaptic contacts ranged from 0.42 to $2.71 \mu \mathrm{m}$, with a mean of $1.09 \mu \mathrm{m}(n=24)$. These values are not statistically different from the length of the unlabeled SLDPs, which were postsynaptic to CR terminals (Mann-Whitney $U$ test, $p>0.1$ ), suggesting that $\mathrm{CR}$ axons terminate on the SLDPs of RS cells.

We then compared the surface areas of SLDPs and the dendritic shafts to assess the possible contribution of the surface area to the specific termination of synapses on SLDPs. From a series of electron micrographs taken from serial sections of the dendrites, the ratio of the dendritic surface area occupied by the SLDPs to that of the shafts was estimated. Two fragments of HRP-labeled RS cell dendrites with a diameter of $\sim 2 \mu \mathrm{m}$ were chosen for this analysis, because these corresponded to the diameters of dendrites on which most CR synapses were formed (Fig. 8). The ratios of the total length of the circumference of SLDPs to that of the shafts for the two dendrites analyzed were 1.69 and 2.16, which are far less than the ratio of synapses on SLDPs and dendritic shafts $(86: 14 \approx 6.1)$.

\section{Location of SLDPs on which CR synapses are formed}

To assess how far the SLDPs are from the soma, the dendritic diameter of HRP-labeled cells was plotted against the distance from the soma. As shown in Figure 8, left panel, the diameter of dendrites decreases as the distance from the soma increases. In addition, by analyzing serial thin sections of CR fibers, we estimated the diameter of parent dendrites of SLDPs that received $\mathrm{CR}$ synapses. The diameter ranged from 0.50 to $5.73 \mu \mathrm{m}$, with a mean of $2.15 \mu \mathrm{m}(n=41)$ (histogram in Fig. 8). A comparison of the diameter of the dendrites from which SLDPs bearing CR synapses emanated (histogram in Fig. 8) with that of HRP-labeled cells indicated that CR fibers formed synapses on SLDPs originating from the proximal dendrites $(<100 \mu \mathrm{m}$ from the soma).

\section{Synapses on somatic membrane}

We also examined synapses on the somatic membrane of HRPlabeled RS cells to compare the synaptic organization (Fig. 9). As shown in Figure $9 A$, the perimeter of the soma was rugged in contour, and synaptic terminals often penetrated into or were engulfed by the soma (Fig. $9 B, C$ ). Although there appeared to be 

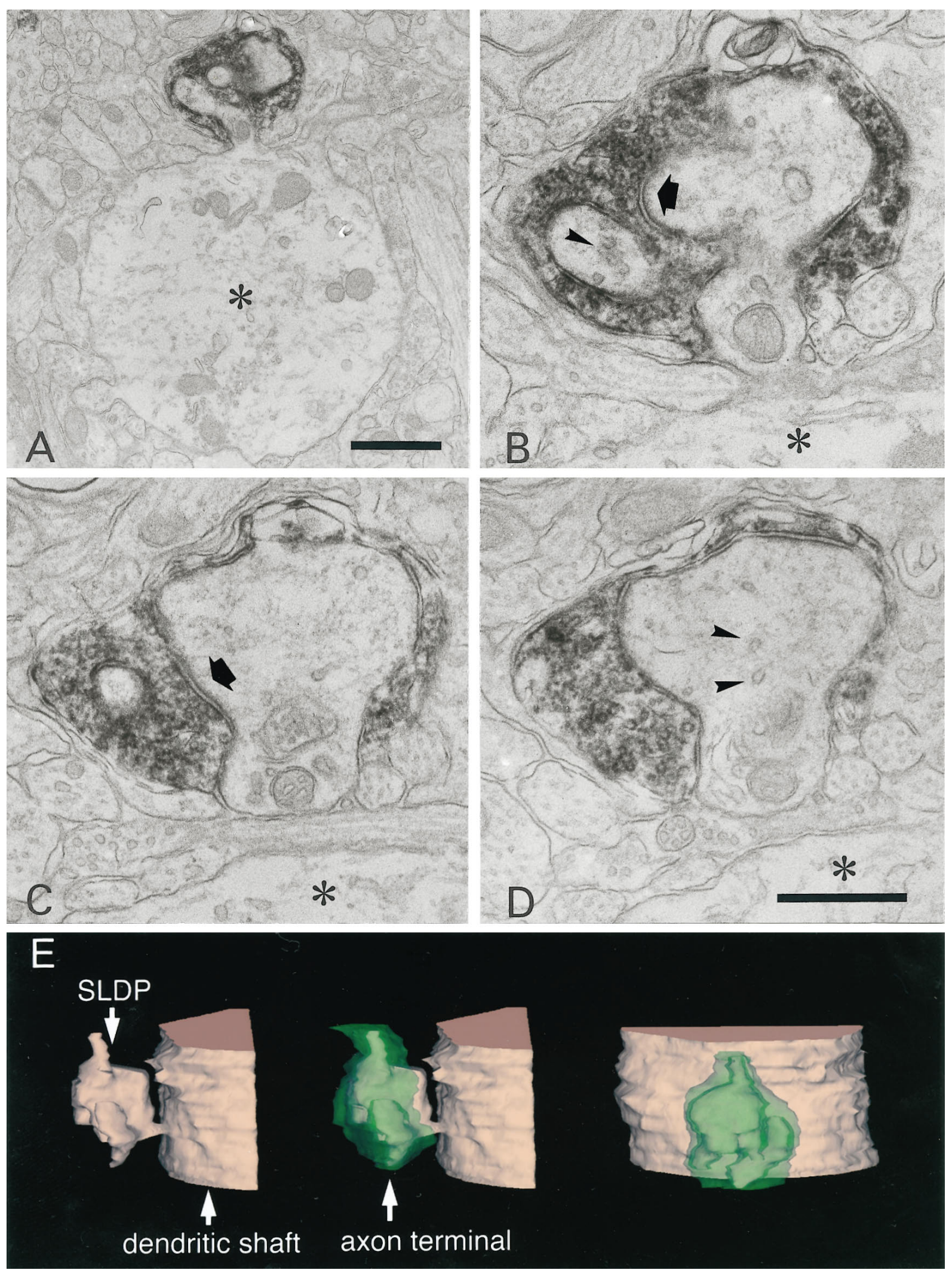

Figure 3. Biocytin-labeled CR axon terminal invaginated by an SLDP. $A-D$, Selected serial electron micrographs of a CR fiber. $A$, Low-magnification electron micrograph of a synapse-bearing dendritic protrusion. $B-D$, High-magnification electron micrographs of serial sections showing an SLDP encapsulated by a CR synaptic ending. The asterisk shows the dendritic shaft. Arrows point to a synapse. Note that many vesicles can be seen within the SLDP (arrowheads). A single CR terminal rarely contacted both the SLDP and the dendrite. E, Three-dimensional reconstruction of CR axon terminal. Profiles shown in tan and green represent the dendrite and the axon terminal, respectively. Other protrusions except for the one shown here were omitted for clarity. The protrusion has multiple branches and invaginates into the synaptic terminal. The left and the center panels show the side view, and the right panel is the top view of the invaginated CR terminal. Scale bars: $A, 1 \mu \mathrm{m} ; B-D, 0.5 \mu \mathrm{m}$. 


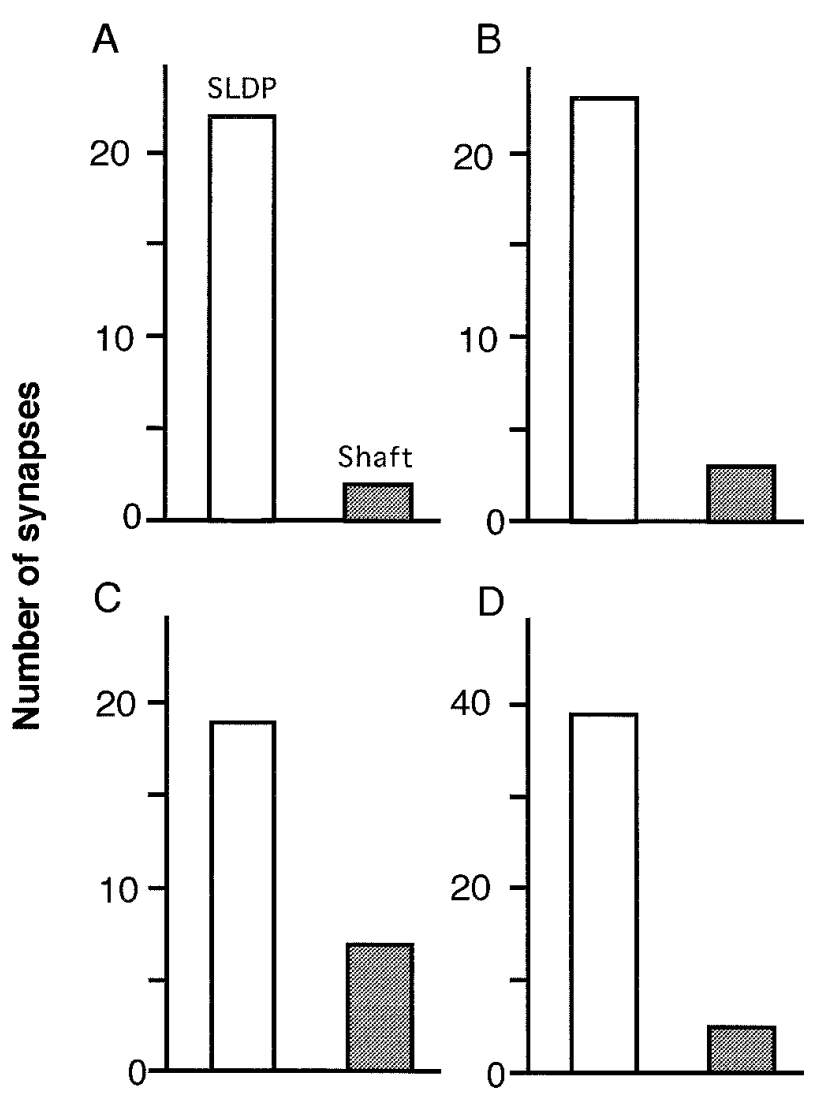

Figure 4. Localization of CR synapses on SLDPs. White and shaded bars represent the number of synapses on SLDPs and dendritic shafts, respectively. $A-C$, Data from individual kittens. A $50-\mu \mathrm{m}$-thick Epon block was cut into $5-\mu \mathrm{m}$-thick sections, and the areas with abundant axonal swellings were selected for thin sectioning. The lengths of the CR fibers analyzed in $A-C$ are $1.1,1.4$, and $2.3 \mathrm{~mm}$, respectively. $D$, Graph from a $50-\mu \mathrm{m}$-thick block directly cut into serial thin sections. In each case most of the synapses are found on SLDPs.

protrusive structures on the somatic membrane, most of them were not associated with synapses, implying that they resulted from synaptic bouton penetration into the somatic membrane. Taking into account of the present results that CR synapses were virtually absent on the somatic membrane, these results raise the possibility that SLDPs play a role in the specific termination of $\mathrm{CR}$ axons on the dendritic membrane.

\section{DISCUSSION}

The present analysis of serially reconstructed CR synapses has revealed that $\mathrm{CR}$ synapses in neonatal cats are formed preferentially on SLDPs of the RN neurons. Such protrusions often invaginated into $\mathrm{CR}$ terminals, forming complex synaptic structures.

\section{The origin of postsynaptic dendritic protrusions}

Although CR fibers terminate on inhibitory interneurons (Katsumaru et al., 1984) in addition to RS cells in adult cats, it is highly likely that the SLDPs postsynaptic to CR fibers included RS cells. In fact, in two cases, we could follow the postsynaptic profiles in serial sections from the SLDPs back to the somata, the diameters of which were $>20 \mu \mathrm{m}$. The fact that the diameter of interneurons is $<20 \mu \mathrm{m}$ even in the adult cat (Katsumaru et al., 1984) suggests that these postsynaptic profiles belong to RS cells. Furthermore, the finding that HRP-labeled RS cells had numer- ous SLDPs with comparable lengths (Figs. 5, 6) is consistent with the view that RS cells are the target of CR fibers. Moreover, our preliminary analysis of the relation of SLDP length with the difference between the length of SLDP head and that of the neck or the ratio between them indicated that there is no difference in morphology between SLDPs postsynaptic to labeled CR fibers and the SLDPs of labeled RS cells. Together, these findings indicate that SLDPs postsynaptic to labeled CR axons included those of RS cells.

\section{Localization of CR synapses on dendritic protrusions}

Serial reconstruction of synapses in the present study unequivocally demonstrated that most of the CR synapses were formed on SLDPs directly emanating from dendritic trunks. This is consistent with the light microscopic observation that dendrites ending in growth cones occurred only rarely. Together, these findings indicate the CR axons terminate on filopodial extensions protruding from the dendritic shaft.

The result that most CR synapses were formed on SLDPs (Fig. 4) indicates that SLDPs may represent the preferred synaptic sites for CR axons. The presence of numerous SLDPs on the dendritic surface (Figs. 5, 6), however, raises the possibility that the localization of CR synapses on SLDPs simply resulted from the larger surface area of the SLDPs compared with that of the dendritic shafts. The estimated ratio between the dendritic surface areas occupied by the SLDPs and the shafts (1.69 and 2.16), however, was much smaller than that of the number of CR synapses on the SLDPs compared with those on the shafts (6.1; Fig. 4). Thus, the preferential localization of CR synapses on SLDPs can only partially be explained by the larger surface area of the SLDP compared with the dendritic shaft and raises the possibility that there are some other mechanisms that promote the preferential termination of CR axons on the SLDPs.

\section{Role of SLDPs in synaptogenesis}

It is likely that the SLDP represents a dynamic feature of the dendrite, because (1) vesicular structures in SLDPs that were associated with smooth endoplasmic reticulum (Figs. 2, 3) were often observed in the growing tips of axons (Peters et al., 1991); (2) invaginated synapses were rarely observed in adult cat RN (Murakami et al., 1982); and (3) spine-like profiles on large-sized $\mathrm{RN}$ neurons appear to decrease in number during the first month of postnatal development (Sadun and Pappas, 1978). The idea that SLDPs dynamically change their structures gains further support from recent time-lapse studies of dissociated neurons and of slice preparations of the hippocampus, which demonstrated that SLDPs are indeed rapidly remodeled (Cooper and Smith, 1992; Dailey and Smith, 1996; Ziv and Smith, 1996).

These findings together with the preferential termination of CR synaptic endings on SLDPs raise the possibility that SLDPs play some role in ongoing synaptogenetic interactions between presynaptic and postsynaptic elements by dynamically changing their structures. Our previous finding that CR axons elaborate arbor during the first month of postnatal development (Higashi et al., 1990), whereas SLDPs decrease in number in the same period (Sadun and Papas, 1978), may be taken to indicate that SLDPs disappear after synaptogenic interactions with CR axons (see below). Moreover, the notion that SLDPs play a role in synaptogenetic interactions is consistent with observations of hippocampal neurons in dissociated culture, in which filopodial extension occurs from the dendritic membrane (Cooper and Smith, 1992; Ziv and Smith, 1996), and the dendritic filopodia seem to initiate 

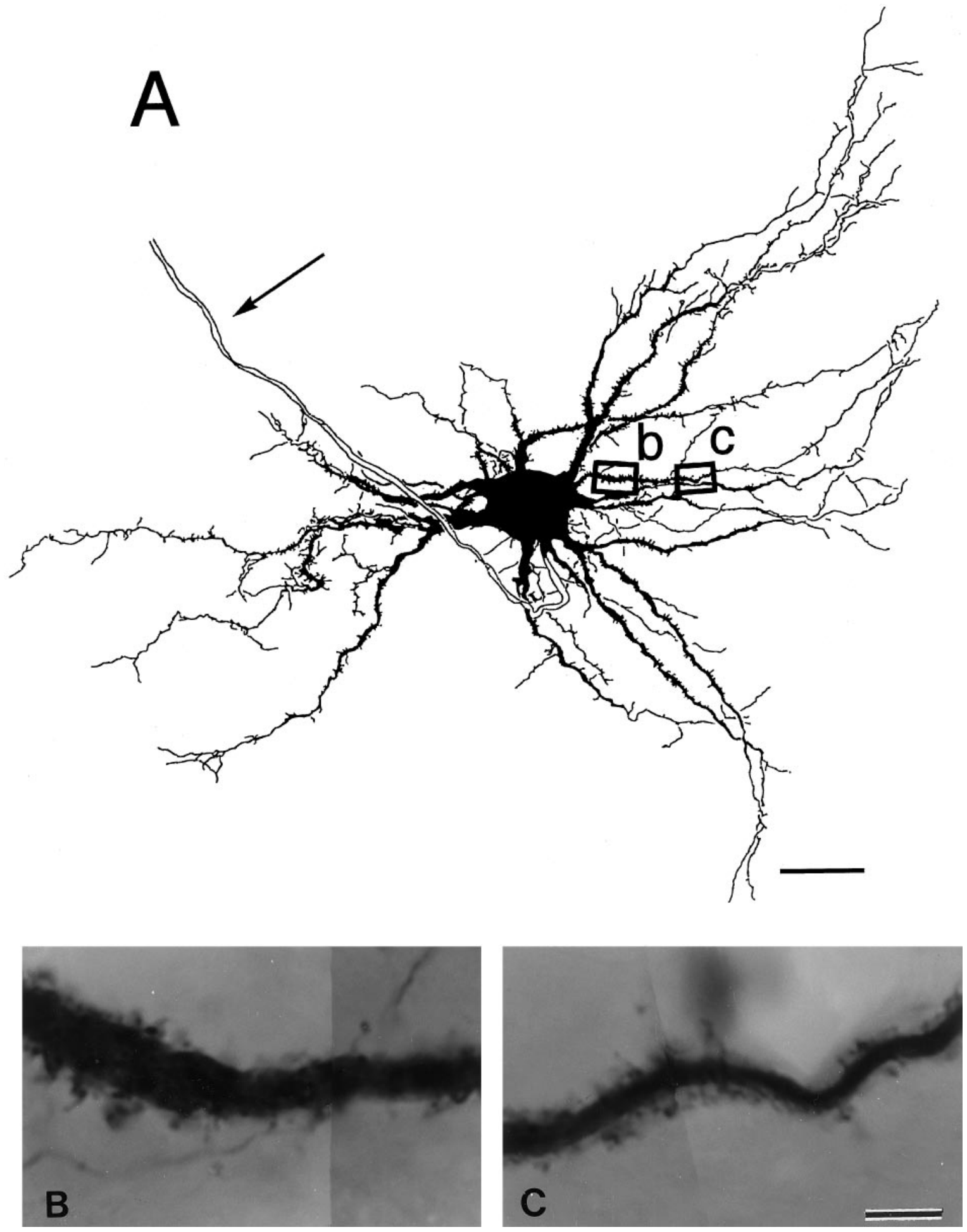

Figure 5. SLDPs emanating from a dendrite of an HRP-labeled RS cell. $A$, Drawing of an RS cell in a newborn kitten. The cell was reconstructed from $50-\mu \mathrm{m}$-thick horizontal sections. The arrow shows the axon. $B, C$, Photomicrographs of the dendritic areas indicated by the two squares and letters in $A$. The photomicrographs in $B$ and $C$ correspond to the areas outlined by $b$ and $c$, respectively. Note that numerous protrusions extend from the dendritic shaft. Scale bars: $A, 50 \mu \mathrm{m} ; B, C, 5 \mu \mathrm{m}$.

physical contact with nearby axons (Cooper and Smith, 1992; Ziv and Smith, 1996). It was also shown that the presumed synaptic site, as indicated by a fluorescent dye that labels synaptic vesicles, occurred in association with dendritic filopodia (Ziv and Smith, 1996), which is consistent with the present results.
What could be the mechanism that allows $\mathrm{CR}$ axons to terminate preferentially on SLDPs? One possibility is that SLDPs attract incoming axons to form synapses (Wong et al., 1992; Papa et al., 1995). In fact, chemoattraction of axons has been shown to play an important role in the guidance of the commissural axons 

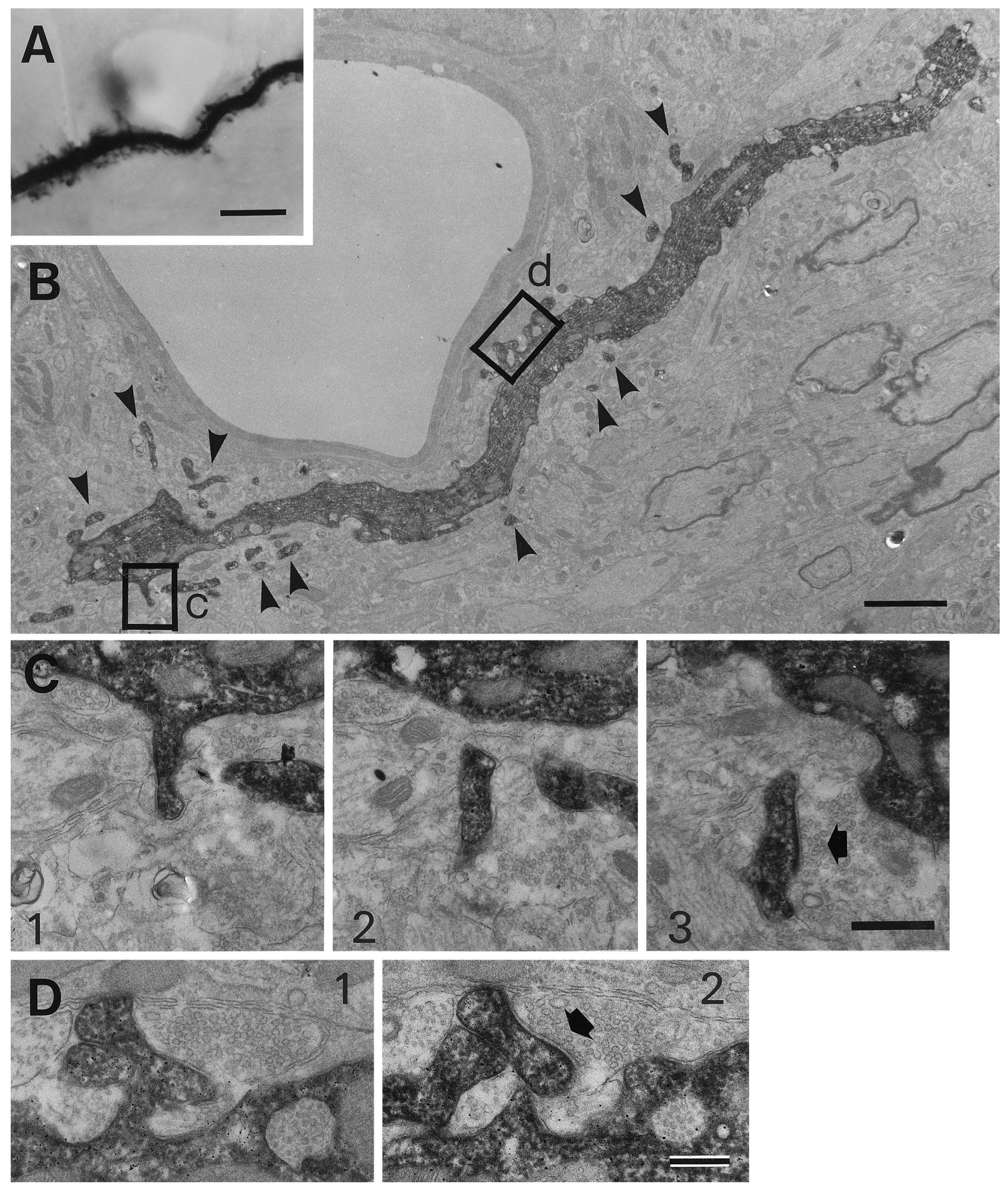

Figure 6. Synapses on SLDPs extending from an HRP-labeled RS cell dendrite. $A$, Photomicrograph of an RS cell dendrite. $B$, Low-magnification electron micrograph of the dendrite shown in $A$. Arrowheads point to labeled SLDPs. $C, D$, SLDPs emanating from the dendritic shaft form synapses (arrows) with unidentified endings. High-magnification electron micrographs of selected serial sections from the two areas outlined by rectangles and letters in $B$. $C$ and $D$ correspond to the areas labeled by $c$ and $d$ in $B$, respectively. Granular profiles in HRP-stained dendrites are reaction products caused by the intensification procedure (see Materials and Methods). Scale bars: $A, 5 \mu \mathrm{m} ; B, 2 \mu \mathrm{m} ; C, 0.5 \mu \mathrm{m} ; D, 0.25 \mu \mathrm{m}$.

of the spinal cord (Tessier-Lavigne et al., 1988; Kennedy et al., 1994) and the hindbrain (Shirasaki et al., 1995; Tamada et al., 1995) during development. On the other hand, a number of studies have indicated that afferents regulate dendritic morphol- ogy (e.g., Rakic, 1975; Kimmel et al., 1977; Caceres and Steward, 1983; Baptista et al., 1994), leading to the hypothesis that incoming axons induce the growth of SLDPs from dendrites (see Vaughn, 1989). Another possibility is that SLDPs dynamically 

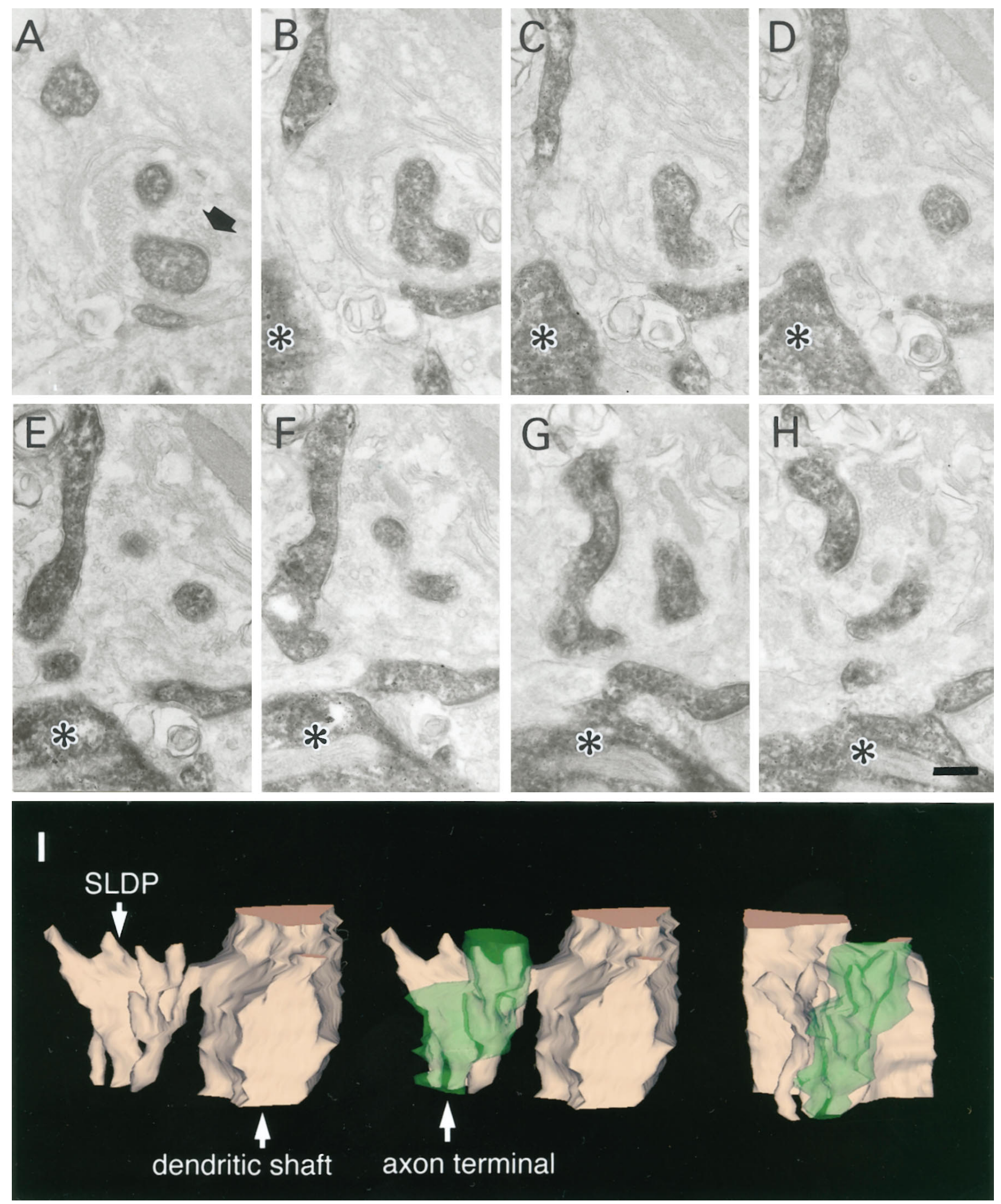

Figure 7. SLDPs of an HRP-labeled RS cell invaginating into an axon terminal. Asterisks show the dendritic shaft from which SLDPs emanate. The black arrow shows the synaptic terminal. $A-H$, Electron micrographs of selected serial sections. $I$, Three-dimensional reconstruction of the presynaptic axon terminal and the SLDP. See legend of Figure 3 for detail. Scale bar, $0.25 \mu \mathrm{m}$.

change their structure (see below), thereby increasing the chance of the incoming CR fibers encountering SLDPs. Elucidation of the role of SLDPs, however, awaits further studies.

The present findings raise another intriguing possibility that
SLDPs contribute to the establishment of synaptic site specificity. Rubrospinal neurons also receive inputs from the interpositus nucleus of the cerebellum (Toyama et al., 1970; Tsukahara et al., 1975), and, in the adult cat, the cerebellar inputs impinge on the 


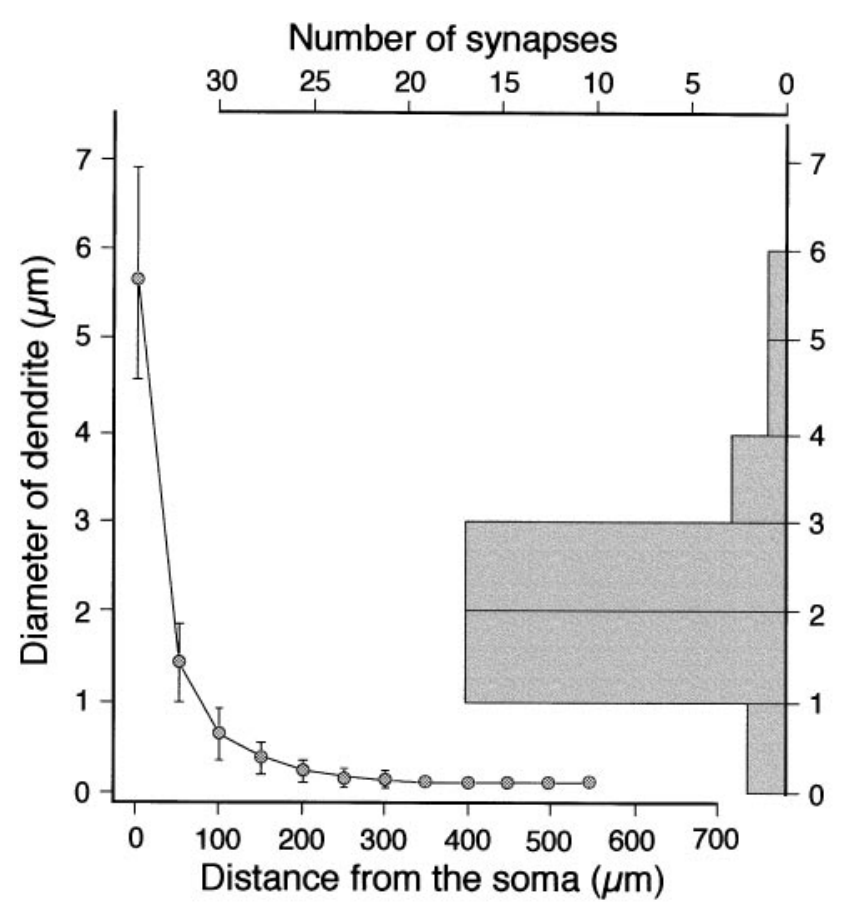

Figure 8. CR synaptic sites on the soma-dendritic membrane of RS cells. The graph to the left shows the diameter of HRP-labeled RS cell dendrites $(n=17)$ plotted against the distance from the soma. The histogram on the right shows the distribution of the diameter of the dendrites from which synapse-bearing SLDPs emanated $(n=41)$. Comparison of the two graphs demonstrates that most of the synapses were located on dendritic regions $<100 \mu \mathrm{m}$ away from the soma.

somatic membrane of RN cells. These inputs arrive in the RN before embryonic day 35 (Song et al., 1995a), far earlier than the entry of cortical inputs, and it is likely that the dendrites of RN cells are only poorly developed at this stage of development. Subsequently, the dendrites may elongate, and SLDPs may develop. The presence of numerous SLDPs at the developmental stage when $\mathrm{CR}$ axons arrive at the RN could facilitate the dendritic termination of CR axons, whereas the virtual absence of filopodial extensions, associated with synapses, on the somatic membrane might provide little chance for somatic termination (but see Povlischock, 1974).

\section{The fate of synapses on SLDPs}

In hippocampal preparations of developing neurons of the rat, motile filopodia decreased in number over extended culture periods, whereas dendritic spines concomitantly increased in number, leading to speculation that dendritic filopodia may be withdrawn into the dendritic shaft or may evolve into spines after synapse formation (Dailey and Smith, 1996; Ziv and Smith, 1996) (also see Hammer et al., 1981; Saito et al., 1992). RS cells in adult cats have spines on their dendrites at distances in excess of 300 $\mu \mathrm{m}$ from the soma (Wilson et al., 1987), and our preliminary electron microscopic study of the adult cat revealed the presence of CR synapses on dendritic spines (Y. Saito and F. Murakami, unpublished observations). These findings suggest that at least a certain proportion of SLDPs transforms into mature spines. However, SLDPs on RN cells decreases in number with maturity of the dendrite (Sadun and Papas, 1978) (C. J. Wilson, F. Murakami, and Y. Saito, unpublished observation), indicating that retraction of SLDPs on RS cells would also occur. The presence of SLDPs
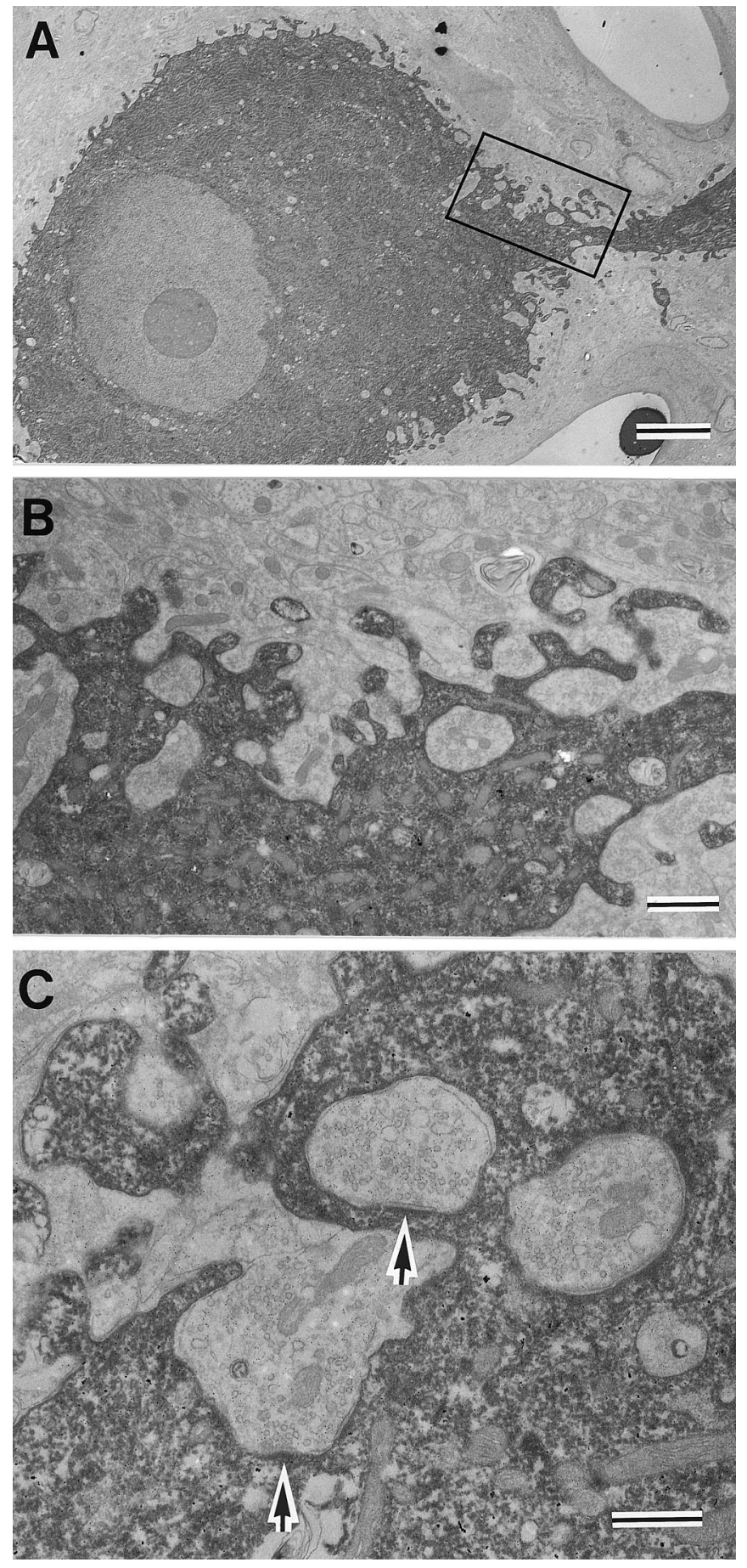

Figure 9. Synapses on the somatic membrane of RS cells. $A$, Electron micrograph of an HRP-filled RS cell. $B$, Higher magnification of the area outlined by the rectangle in $A$. Note the presence of inclusions of synaptic endings in the soma. $C$, Synapses formed by terminals included in the soma (arrows). Similar inclusions were observed in nonstained soma, indicating that these are not artifacts of the HRP injection (not shown). Scale bars: $A, 5 \mu \mathrm{m} ; B, 1 \mu \mathrm{m} ; C, 0.5 \mu \mathrm{m}$.

without synapses (data not shown) (also see Papa et al., 1995) implies that those that failed to make synapses eventually retract.

In conclusion, in the present in vivo study, we demonstrated what cannot be shown with in vitro techniques: preferential termination of synapses on spine-like dendritic protrusions. Taken together with previous in vitro studies that demonstrated dynam- 
ical features of SLDPs, our results suggest that SLDPs may commonly play an active role in synaptogenic interactions.

\section{REFERENCES}

Baptista CA, Hatten ME, Blazeski R, Mason CA (1994) Cell-cell interactions influence survival and differentiation of purified Purkinje cells in vitro. Neuron 12:243-260.

Blue ME, Parnavelas JG (1983) The formation and maturation of synapses in the visual cortex of the rat. I. Quantitative analysis. J Neurocytol 12:599-616.

Boothe RG, Greenough WT, Lund JS, Wrege K (1979) A quantitative investigation of spine and dendrite development of neurons in visual cortex (area 17) of Macaca nemestrina monkeys. J Comp Neurol 186:473-490.

Caceres A, Steward O (1983) Dendritic reorganization in the denervated dentate gyrus of the rat following entorhinal cortical lesions: a Golgi and electron microscopic analysis. J Comp Neurol 214:387-403.

Cooper MW, Smith SJ (1992) A real-time analysis of growth cone-target cell interactions during the formation of stable contacts between hippocampal neurons in culture. J Neurobiol 23:814-828.

Dailey ME, Smith SJ (1996) The dynamics of dendritic structure in developing hippocampal slices. J Neurosci 16:2983-2994.

Dodd J, Jessel TM (1988) Axon guidance and the patterning of neural projections in vertebrates. Science 242:692-699.

Dvergsten CL, Hull CD, Levine MS, Adinolfi AM, Buchwald NA (1986) Postnatal differentiation and growth of cat entopeduncular neurons. A transient spiny period associated with branch elongation. Dev Brain Res 24:239-251.

Garey LJ, Saini KD (1981) Golgi studies of the normal development of neurons in the lateral geniculate nucleus of the monkey. Exp Brain Res 44:117-128.

Goodman CS (1996) Mechanisms and molecules that control growth cone guidance. Annu Rev Neurosci 19:341-377.

Goodman CS, Shatz CJ (1993) Developmental mechanisms that generate precise patterns of neuronal connectivity. Neuron 10:77-98.

Hammer Jr RP, Lindsay RD, Scheibel AB (1981) Development of the brain stem reticular core: an assessment of dendritic state and configuration in the perinatal rat. Dev Brain Res 1:179-190.

Hayes BP, Roberts A (1973) Synaptic junction development in the spinal cord of an amphibian embryo: an electron microscope study. Z Zellforsch Mikrosk Anat 137:251-269.

Higashi S, Yamazaki M, Murakami F (1990) Postnatal development of crossed and uncrossed corticorubral projections in kitten: A PHA-L study. J Comp Neurol 299:312-326.

Hinds JW, Hinds PL (1976) Synapse formation in the mouse olfactory bulb. II. Morphogenesis. J Comp Neurol 169:41-62.

Jacobson M (1991) Developmental neurobiology, Ed 3. New York: Plenum.

Juraska JM, Fifkova E (1979) An electron microscope study of the early postnatal development of the visual cortex of the hooded rat. J Comp Neurol 183:257-268.

Katsumaru H, Murakami F, Wu J-Y, Tsukahara N (1984) GABAergic intrinsic interneurons in the red nucleus of the cat demonstrated with combined immunocytochemistry and anterograde degeneration methods. Neurosci Res 1:35-44.

Kennedy TE, Serafini T, de la Torre JR, Tessier-Lavigne M (1994) Netrins are diffusible chemotropic factors for commissural axons in the embryonic spinal cord. Cell 78:425-435.

Kosar E, Fujito Y, Murakami F, Tsukahara N (1985) Morphological and electrophysiological study of sprouting of corticorubral fibers after lesions of the contralateral cerebrum in kitten. Brain Res 347:217-224.

Kimmel CB, Schabtach E, Kimmel RJ (1977) Developmental interactions in the growth and branching of the lateral dendrite of Mauthner's cell (Ambystoma mexicanum). Dev Biol 55:244-259.

Kunkel DD, Westrum LE, Bakay RAE (1987) Primordial synaptic structures and synaptogenesis in rat olfactory cortex. Synapse 1:191-201.

Lund JS, Boothe RG, Lund RD (1977) Development of neurons in the visual cortex (area 17) of the monkey (Macaca nemestrina): a Golgi study from fetal day 127 to postnatal maturity. J Comp Neurol 176:149-188.

Morest DK (1969) The growth of dendrites in the mammalian brain. Z Anat Entwicklungsgesch 128:290-317.

Murakami F, Higashi S (1988) Presence of crossed corticorubral fibers and increase of crossed projections after unilateral lesions of the cerebral cortex of the kitten: a demonstration using anterograde transport of Phaseolus vulgaris leucoagglutinin. Brain Res 447:98-108.

Murakami F, Katsumaru H, Saito K, Tsukahara N (1982) A quantitative study of synaptic reorganization in red nucleus neurons after lesion of the nucleus interpositus of the cat: an electron microscopic study involving intracellular injection of horseradish peroxidase. Brain Res 242:41-53.

Murakami F, Katsumaru H, Wu J-Y, Matsuda T, Tsukahara N (1983) Immunocytochemical demonstration of GABAergic synapses on identified rubrospinal neurons. Brain Res 267:357-360.

Murakami F, Etoh M, Kawato M, Oda Y, Tsukahara N (1986) Synaptic currents at interpositorubral and corticorubral excitatory synapses measured by a new iterative single-electrode voltage-clamp method. Neurosci Res 3:590-605.

Murakami F, Higashi S, Yamazaki M, Tamada,A (1991a) Lesioninduced establishment of the crossed corticorubral projections in kittens is associated with axonal proliferation and topographic refinement. Neurosci Res 12:122-139.

Murakami F, Saito Y, Higashi S, Oikawa H (1991b) Synapses formed by ectopic corticofugal axons: an electron microscopic study of crossed corticorubral projections in kittens. Neurosci Lett 131:49-52.

Murakami F, Kobayashi Y, Uratani T, Tamada A (1993) Individual corticorubral neurons project bilaterally during postnatal development and following early contralateral cortical lesions. Exp Brain Res 96:181-193.

Nakamura Y, Mizuno N (1971) An electron microscopic study of the interposito-rubral connections in the cat and rabbit. Brain Res 35:283-296.

Nakamura Y, Mizuno N, Konishi A (1978) A quantitative electron microscope study of cerebellar axon terminals on the magnocellular red nucleus neurons in the cat. Brain Res 147:17-27.

Papa M, Bundman MC, Greenberger V, Segal M (1995) Morphological analysis of dendritic spine development in primary cultures of hippocampal neurons. J Neurosci 15:1-11.

Peters A, Palay SL, Webster HdF (1991) The fine structure of the nervous system. New York: Oxford UP.

Phelps PE, Adinolfi AM, Levine MS (1983) Development of the kitten substantia nigra: a rapid Golgi study of the early postnatal period. Dev Brain Res 10:1-19.

Pizzini G, Tredici G, Miani A (1975) Corticorubral projection in the cat. An experimental electronmicroscopic study. J Submicr Cytol 7:231-238.

Povlishock JT (1974) The presence of perisomatic processes during maturation of the hypoglossal, vagal and red nuclei of the rat. Brain Res 82:272-278.

Rakic P (1975) Role of cell interaction in development of dendritic patterns. Adv Neurol 12:117-134.

Ramoa AS, Campbell G, Shatz CJ (1988) Dendritic growth and remodeling of cat retinal ganglion cells during fetal and postnatal development. J Neurosci 8:4239-4261.

Sadun AA, Pappas GD (1978) Development of distinct cell types in the feline red nucleus: a Golgi-Cox and electron microscopic study. J Comp Neurol 182:315-366.

Saito Y, Murakami F, Song W-J, Okawa K, Shimono K, Katsumaru H (1992) Developing corticorubral axons of the cat form synapses on filopodial dendritic protrusions. Neurosci Lett 147:81-84.

Scheibel ME, Davies TL, Scheibel AB (1973) Maturation of reticular dendrites: loss of spines and development of bundles. Exp Neurol 38:301-310.

Shirasaki R, Tamada A, Katsumata R, Murakami F (1995) Guidance of cerebellofugal axons in the rat embryos: directed growth toward the floor plate and subsequent elongation along the longitudinal axis. Neuron 14:961-972.

Song W-J, Kanda M, Murakami F (1995a) Prenatal development of cerebrorubral and cerebellorubral projections in cats. Neurosci Lett 200:41-44.

Song W-J, Okawa K, Kanda M, Murakami F (1995b) Perinatal development of action potential propagation in cat rubrospinal axons. J Physiol (Lond) 488:419-426.

Tamada A, Shirasaki R, Murakami F (1995) Floor plate chemoattracts crossed axons and chemorepels uncrossed axons in the vertebrate brain. Neuron 14:1083-1093.

Tessier-Lavigne M, Placzek M, Lumsden A, Dodd J, Jessel TM (1988) 
Chemotropic guidance of developing axons in the mammalian central nervous system. Nature 336:775-778.

Toyama K, Tsukahara N, Kosaka K, Matsunami K (1970) Synaptic excitation of red nucleus neurons by fibers from interpositus nucleus. Exp Brain Res 11:187-198.

Tsukahara N, Kosaka K (1968) The mode of cerebral excitation of red nucleus neurons. Exp Brain Res 5:102-117.

Tsukahara N, Murakami F, Hultborn H (1975) Electrical constants of neurons of the red nucleus. Exp Brain Res 23:49-64.

Tsukahara N, Fujito Y, Kubota M (1983) Specificity of the newly-formed corticorubral synapses in the kitten red nucleus. Exp Brain Res 51:45-56.

van den Pol AN, Gorse T (1986) Synaptic relationship between neurons containing vasopressin, gastrin-releasing peptide, vasoactive intestinal polypeptide, and glutamate decarboxylase immunoreactivity in the suprachiasmatic nucleus: dual ultrastructural immunocytochemistry with gold-substituted sliver. J Comp Neurol 252:507-521.

Vaughn JE (1989) Fine structure of synaptogenesis in the vertebrate central nervous system. Synapse 3:255-285.

Villablanca JR, Olmstead CE, Sonnier BJ, McAlister JP, Gomez-Pinilla F (1982) Evidence for a crossed corticorubral projection in cats with one cerebral hemisphere removed neonatally. Neurosci Lett 33:241-246.

Wilson CJ, Murakami F, Katsumaru H, Tsukahara N (1987) Dendritic and somatic appendages of identified rubrospinal neurons of the cat. Neuroscience 22:113-130.

Wong ROL, Yamawaki RM, Shatz CJ (1992) Synaptic contacts and the transient dendritic spines of developing retinal ganglion cells. Eur J Neurosci 4:1387-1397.

Ziv NE, Smith SJ (1996) Evidence for a role of dendritic filopodia in synaptogenesis and spine formation. Neuron 17:91-102. 